\title{
Kirche und Publizistik. Ein Rückblick auf Erfolge und Mißerfolge in den letzten Jahrzehnten ${ }^{1}$
}

\author{
von Otto B. Roegele
}

Schon auf dem Mainzer Katholikentag von 1948, dem 72. der ganzen Reihe seit 1848 und ersten Katholikentag nach dem II. Weltkrieg, meldete sich das Thema "Publizistenausbildung' gebieterisch zu Wort. Ein selbständiger Programmpunkt war dafür zwar nicht vorgesehen; aber in der Diskussion der Arbeitsgemeinschaft "Katholische Publizistik am 02.09.1948 nahm es eine beherrschende Stellung ein. Über das Wie und Wo gab es Meinungsverschiedenheiten wie eh und je, aber daß etwas geschehen müsse, um den Mangel an religiös wie beruflich qualifizierten Kräften zu beheben, stand für alle fest.

Schließlich wurde die eben gegründete, Gesellschaft katholischer Publizisten Deutschlands“ beauftragt, "unverzüglich mit der Vorbereitung von Kursen für den Nachwuchs zu beginnen, in denen jüngere Journalisten einige Tage mit Fachleuten der Theorie und Praxis über bestimmte Themen- und Fragenkomplexe arbeiten sollen, um so über den Stand der Probleme unterrichtet zu werden".2

Es war also nicht nur ihr eigenes Anliegen, sondern geschah auch in Erfüllung dieses Auftrages, wenn die neue ,Gesellschaft' in der Folgezeit immer wieder Versuche unternahm, Journalisten und solche, die es werden wollten, dazu anzuleiten, wie sie gute Christen und gute Journalisten zugleich sein könnten.

Das mag heute in manchen Ohren etwas altmodisch klingen, schließlich leben wir im Zeitalter des Redaktronikers und des Infotainers, und wer in heutigen Fachzeitschriften blättert, muß annehmen, daß Datensysteme und Meßapparate für Einschaltquoten viel wichtiger geworden sind als Inhalte. Aber damals war es keineswegs altmodisch und heute mag es auch an den Ohren liegen, wenn es so klingt. Noch immer heißt die Aufgabe kirchlicher Bemühungen um den Nachwuchs in der Publizistik: der engagierte Christ von beruflicher Kompetenz.

Prof. Dr. Dr. Otto B. Roegele ist Mitherausgeber der Wochenzeitung Rheinischer Merkur' und Vorsitzender des 'Instituts zur Förderung publizistischen Nachwuchses e. V.' in München.

I Vortrag aus Anlaß des 25jährigen Bestehens des 'Instituts zur Förderung publizistischen Nachwuchses e. V.' vom 14.11.1993 in Ludwigshafen.

2 "Der Christ in der Not der Zeit". Protokollband des Mainzer Katholikentages, Paderborn 1949, 179. 
Dabei war es nicht so, daß die Gesellschaft beim Nullpunkt hätte anfangen müssen. Überall, wo katholische Journalisten und Verlage in jenen Jahren zusammenkamen, beschäftigten sie sich mit der Frage nach der Förderung des Nachwuchses, ob das die Tagung der ,Katholischen Laienwerke' (März 1948 in Limburg) oder die ,Internationale Werkwoche katholischer Publizisten' in Walberberg bei Bonn (26.04. bis 02.05.1948) war, ob es der ,Sachausschuß für Presse und Schrifttum' des „Diözesankomitees der Katholikenausschüsse der Erzdiözese Köln' war oder die Verleger, die sich in Gruppen und Gremien trafen - alle sprachen von ihrer Bereitschaft, etwas für den Nachwuchs zu tun. Einzelheiten brauchen nicht aufgeführt zu werden, das Wichtigste kann in einer Münchner Magister-Arbeit von Petra Kuhbier aus dem Jahre 1985 nachgelesen werden.

Zwei Namen, zwei über längere Zeit wirkende Unternehmungen möchte ich zuerst nennen: Pater Dr. Heinrich Jansen Cron SJ, der in seiner Person bereits ein ganzes, Institut zur Förderung des schreibenden Nachwuchses' darstellte, mit dessen Zeitschrift ,Leuchtturm' viele von uns Älteren das Schreiben für die Öffentlichkeit erst gelernt haben, unter liebevoll-unbarmherziger Anleitung und manche noch unter den komplizierenden Bedingungen des nationalsozialistischen Regimes, und Dr. Hermann Boventer, der in Zusammenarbeit der von ihm geleiteten ,Thomas Morus-Akademie' mit dem ,Zentralkomitee der deutschen Katholiken' über Jahre hinweg die ,Bensberger Kurse' als Berufs- und Erwachsenenbildungsangebot gegen manche Hindernisse aufrechterhielt.

Das Gedächtnis des deutschen Katholizismus ist nicht sonderlich gut entwickelt, und das hängt wohl auch mit einem gewissen Mangel an kollektiver Dankbarkeit zusammen. Deshalb nenne ich noch Dr. Hans Suttner, dessen man sich allenfalls noch im Zusammenhang mit der Gründung von ,Publik' erinnert. Wer weiß noch, daß er in seiner Denkschrift ,Katholische Presse in Deutschland', die er zwecks schnellerer Verbreitung listigerweise mit dem Vermerk "Vertraulich" und einer Zählnummer versah, im Spätsommer 1965 ein sehr viel umfassenderes Projekt entworfen hatte: nämlich die Zusammenfassung aller publizistischen Anstrengungen des deutschen Katholizismus in einem ,Institut Publizistik‘. In diesem Zusammenhang sollte auch die große katholische Wochenzeitung entstehen, aber sie nahm in seinen Vorschlägen eine viel geringere Position ein als etwa die Anregungen zu Personalfragen: Dazu gehörte die Anlage einer Kartei aller katholischen Medien-Mitarbeiter, die "Bildung eines schlagkräftigen Teams junger Publizisten", einer Eingreiftruppe für den Meinungskampf sozusagen, die Gründung einer "Journalistenschule neuen Stils", die systematisch betriebene Talentsuche, die Heranziehung wissenschaftlicher Analysen für die praktische Verlags- und Redaktionsarbeit; und dies alles eingebunden in und ständig fruchtbar gemacht für eine das ganze Land erfassende "Schulungsarbeit", mit Priorität für die weltanschauliche, religiöse, apologetische Schulung und mit dem wichtigen Hinweis, daß man 
Talente nicht nur suchen, finden und fördern, sonder auch "halten“ müsse.

Was Hans Suttner damals veranlaßte, alles dies in einem Institut unterzubringen, war seine Ansicht, daß die immer wieder neu ansetzenden, im Lande verstreuten, einander jeweils nur partiell zur Kenntnis nehmenden Aktivitäten dem großen Bedarf nicht gerecht werden konnten und einen nicht zu verantwortenden Aufwand an Mühe verursachten. Auf die Dauer und rationell wirksam werden - das konnte nur eine ständige Einrichtung mit Überblick.

Diese Erfahrung drängte sich in jenen Jahren auch anderen auf. Ich weiß nicht mehr, wie viele Denkschriften, Eingaben und Projekte ich selbst der Deutschen Bischofskonferenz zugeleitet oder mitunterzeichnet habe, sei es als Vorsitzender der 'Gesellschaft katholischer Publizisten', sei es vom Boden des ,Zentralkomitees der deutschen Katholiken' aus. Im Januar 1967 wurde es jedenfalls zum ersten Mal wirklich konkret, als die ,Publizistische Kommission der Deutschen Bischofskonferenz' einen neuen "Unterausschuß "Förderung des publizistischen Nachwuchses“" nach Bad Godesberg einberief und ein Konvolut von Papieren unterschiedlicher Herkunft zur Grundlage von weiteren Beratungen machte. Das Erstaunlichste bei diesem Vorgang war, daß nun alles sehr rasch gehen sollte. In ersten Umrissen zeichnete sich das Institut ab, wie es am 11.10.1968 in München ins Leben trat: das Modell einer studienbegleitenden Ausbildung in zwei Dimensionen: Religion und Beruf.

Es ist in meinen Augen eine Bestätigung für das Walten der Vorsehung (und für die fehlende Erklärungskraft des Begriffs Zufall), daß die Gründung des Instituts noch rechtzeitig vor der Würzburger Synode zustandekam. Die Institutspläne wären sonst unweigerlich in dem Strudel von Enttäuschung und Depression untergegangen, den das Scheitern von "Publik' hervorrief und die das auslösende Ereignis an Tiefen- und Dauerwirkung weit überragten.

Man sollte nicht so rasch vergessen: Auf der Würzburger Synode ist es der Publizistik als Gegenstand und der öffentlichen Meinung als deren Wirkungsfeld nicht gut ergangen. Ich weiß, daß das eine Feststellung ist, die nicht gerne akzeptiert wird. Die Synode ist zu einer Art Ikone geworden, bei deren Verehrung die Frage nach Ähnlichkeit mit der historischen Realität nicht gestellt werden darf. Um der Wahrheit willen muß jedoch festgehalten werden: Die Publizistik war weder in den Vorbereitungsarbeiten noch im Programm als selbständiger Fachbereich durch eine eigene Kommission präsent, obwohl man die praktische Seite sehr wohl einbezogen hatte: es gab eine tüchtig arbeitende Pressestelle mit kompletter Information durch Dokumente und Pressekonferenzen, was den Verzicht umso unbegreiflicher erscheinen läßt. 
Das Thema ,Publik und sein Scheitern' mußte durch einen Antrag der Synodalen sozusagen erzwungen werden; die auf der Sondersitzung am 11.05.1972 geleistete "Trauerarbeit" gelang nur unvollkommen, um nicht zu sagen: gar nicht. Schon die erste Voraussetzung, die Bereitschaft zur Einsicht in die Ursachen des Todes war nicht gegeben. Es blieben Emotionen und Schuldzuweisungen, die sich in Ressentiments dauerhaft niederschlugen. Wäre damals nicht das improvisierte "Sofortprogramm" von Bischof Moser vorgetragen worden, hätte die Sondersitzung nur verderbliche Folgen gezeitigt. So konnte immerhin erreicht werden, daß der Kommission VI („Erziehung, Bildung, Information“) der Auftrag erteilt wurde, anstelle einer ursprünglich vorgesehenen, dann aber gestrichenen Vorlage für einen Synoden-Beschluß ein "Kommissionspapier" auszuarbeiten. Die Verhandlungen der Synode waren bereits weit fortgeschritten, in beschwerlicher Randlage schleppten sich die Beratungen über dieses "Papier" hin, niemandem war der Spätling so recht willkommen, das Ergebnis wurde in einem etwas konfusen Verfahren verabschiedet und als Dokument zweiten Ranges in einem „Ergänzungsband" der "offiziellen Gesamtausgabe der SynodenDokumente ${ }^{\text {/3 }}$ veröffentlicht.

Man mag über die kirchengeschichtliche Bedeutung und den dauernden Nutzen der Würzburger Synode unterschiedlich denken; ein Urteil muß ohnehin späteren Generationen überlassen bleiben. Für mich steht jedenfalls fest, daß die Synode, indem sie dem Gesamtbereich ,Öffentliche Meinung' (den Medien, die sie in Gang halten; den Katholiken, die von ihnen beeinflußt werden; denen, die für sie arbeiten; dem ganzen machtvollen Apparat der "Massenführung", dem Problem der zunehmenden Sekundärwahrnehmung, der Vorspiegelung einer manipulierten Welt) von vornherein keinen eigenen angemessenen Platz in ihren Beratungen zubilligte, sich als eine Unternehmung von geringem Weitblick und folgenschwerer Selbstbeschränkung erwies. Wer bei uns versucht ist, römischen Instanzen den Vorwurf einer Vernachlässigung dieses ganzen Bereichs zu machen, sollte zuerst im eigenen Hause Nachschau halten. Diese Erfahrungen begründen auch meine Skepsis gegenüber dem in den letzten Jahren immer wieder propagierten Gedanken, die Kirchenversammlung von Würzburg zu wiederholen, um die Sache der Kirche in der modernen Welt voranzubringen. Zwei Jahre lang waren die Aktivisten der kirchlichen Arbeit auf die eine oder andere Weise mit der Synode beschäftigt und von ihrer Tätigkeit an Ort und Stelle, in ihrem eigentlichen Arbeitsfeld, mehr oder weniger stark abgehalten. Währenddessen hat die „weltliche Welt" sich unbekümmert weiterbewegt. Es verlangt mich nicht nach einer Wiederholung solcher Erfahrungen, auch nicht unter der Devise "Gespräche sind immer nützlich".

Das bischöfliche „Sofortprogramm“ verhieß Förderung des Nach-

3 Freiburg i. Br. 1977. 
wuchs-Instituts, und daran fehlte es auch nicht. Unter der Leitung von Pater Dr. Wolfgang Seibel SJ, dem Chefredakteur der ,Stimmen der Zeit', in engagierter Mitwirkung von Dr. Wilfried Schwedler, konnte eine sehr persönlich geprägte und effiziente Stipendiaten-Förderung aufgebaut werden, die in manchen Zügen fast an die MeisterSchüler-Beziehung der japanischen Journalisten-Ausbildung erinnert. Es war die „heroische Zeit der Frühe" für das Institut, nicht ohne Kämpfe und kritische Situationen, denn es waren ja auch die Jahre der studentischen Rebellion.

Erlauben Sie mir, zu Aufgaben und Struktur des Instituts einige persönliche Anmerkungen zu machen, die zeigen, daß das Institut nicht als statisches Gebilde zu verstehen ist, sondern als ein in der Zeit und mit ihr lebendes Wesen, das auf Fragen, die von außen kommen, ebenso Antworten suchen muß wie auf Fragen, die es sich selbst stellt.

Aus dem Familienbetrieb der Anfangszeit mit allen seinen Vorzügen und Eigenheiten ist längst ein Manufakturbetrieb geworden, in den Arbeitsteilung, Verwaltung, Organisation, Planung von Raum und Zeit, Effizienzkontrolle und Haushaltsprüfung eine wachsende Rolle spielen. Viele neue Aufgaben sind hinzugekommen, die nicht bewältigt werden könnten, wenn es sich nicht so gefügt hätte, daß die Studio-Anlagen in Ludwigshafen in die Obhut des Instituts übergeben wurden. Dr. Anton Magnus Dorn hat hier sein Reich aufgebaut, das viele Nutzungs- und Kooperationschancen eröffnete. Mit dem Wegfall der Grenzen zum bisher kommunistisch beherrschten Europa, das die DDR einschloß, sind neue Herausforderungen aufgetaucht, denen sich das Institut nicht entziehen kann, wenn es seine Zielsetzung nicht verleugnen will. Eine entschlossene, zugleich vorsichtige Expansion war nötig, um eine Soforthilfe für die "neuen Bundesländer", dann auch eine "Erste Hilfe" für Journalisten aus osteuropäischen Nachbarländern zu ermöglichen. Auch hier hat eine Fügung uns geholfen, als wir Pater Gerhard Eberts gerade im richtigen Augenblick für uns gewinnen konnten.

Über der geographischen und strukturellen Expansion darf nicht versäumt werden, daß wir auch die religiös-ethische Dimension so verstärken, daß sie die neugewonnen Räume auszufüllen erlaubt.

$\mathrm{Zu}$ den Kennzeichen des Instituts gehört, daß Christsein und Journalistsein nicht als getrennte Welten erfahren werden, die sich zufällig in ein und demselben Menschen treffen. Gewiß, der Journalist, der seinen Beruf als Christ ausübt, wendet keine anderen Handwerksregeln an als der nichtchristliche Kollege, der seinen Beruf ernstnimmt. Aber sein ganzes Leben steht in einem weiteren Seinszusammenhang, es ist offen nach oben. Der katholische Journalist hat ja nicht nur innerweltliche Motive, er versteht sich als ein Gerufener, der dem, der ihn gerufen hat, Rechenschaft schuldet. 
Das stiftet das Bewußtsein einer Verantwortung, die weiter reicht und tiefer gründet, auch unentrinnbarer ist als alle Verantwortungen gegenüber dem Arbeitgeber, dem Gesetzbuch, dem Ehrenkodex der Zunft, ja sogar dem Publikum, dessen Vertrauen man besitzt. Wer seine Berufspflichten in Einklang mit der Stimme seines Gewissens erledigen kann, wer da nicht nur Mühe, Strapaze und Verschleiß, sondern auch Erfüllung findet, hat allen Grund, dankbar zu sein. Er erwirbt sich dann nicht den Himmel am Sonntag und das tägliche Brot am Werktag, sondern er dient Gott auch im Beruf. Hier gewinnt das Wort von der ,Fronarbeit' einen neuen Sinn: Herrenarbeit, Arbeit für den Herrn. Niemand kann dem Augenblick, da die persönliche Rechenschaft gefordert wird, ohne Zittern entgegensehen. Jeder Lebensweg führt ja auf einen Richter zu, der alles weiß, der in die Falten des Herzens blickt - und von diesem Endpunkt her muß auch das Verlangen nach einer Ethik für Journalisten gesehen werden.

$\mathrm{Zu}$ den erfreulichen Entwicklungen unserer Gegenwart gehört es, $\mathrm{da} ß$ in vielen Bereichen, die bisher mit Rechenstift und "Sachwissen" auszukommen glaubten, der Ruf nach einer speziellen Ethik ertönt. Wenn wirtschaftswissenschaftliche Fakultäten Lehrstühle für Wirtschaftsethik einrichten, ist das ein deutlich vernehmbares Signal dafür, $\mathrm{da} ß$ sich ein Wandel vollzieht. Die Diskussion, was Ethik als Gegenstand der kommunikationswissenschaftlichen Betrachtung bedeutet, ist noch im Gange; wir dürfen das Ergebnis in Ruhe abwarten. Es kommt für uns mehr darauf an, daß Rang und Wahrnehmung der ethischen Normen im Berufshandeln der Medienschaffenden im weitesten Sinne fester in den Blick genommen und in der Ausbildung berücksichtigt werden.

Diese Aufgabe lege ich besonders Herrn Prof. Dr. Josef Innerhofer ans Herz, der nun das Institut leitet und, wie ich hoffe, über die Jahrtausendschwelle hinüberführen wird. Daß wir ihn gewinnen konnten, rechne ich zu den großen Glücksfällen des Instituts und meines Lebens. Ich wünsche ihm und seiner Equipe, daß es ihnen gelingt, den Geist des Anfangs zu bewahren und weiterzugeben, in dem so viele junge Menschen die Ausrüstung für ihren Beruf gefunden haben: katholisch (und das heißt immer auch: apostolisch, notfalls sogar apologetisch), wahrheitsoffen, weltneugierig, menschenfreundlich, mit Freude am Beruf, am Jungsein, am Erfolg, am Leben überhaupt am Leben in der Kirche und mit der Kirche.

SUMMARY: Church and Communications. A retrospective on successes and failures in the recent decades

The author gives a personal retrospective on the history of the relation of the Church and the media in the post-war Federal Republic of Germany. He depicts impressions of the complicate history of the formation of the ecclesiastical "Institut zur Förderung publizistischen Nachwuchses e. V." 
(institution for support and formation of young catholic journalists) and the fruitless attemps of the Catholic Church to establish a big weekly newspaper called "Publik". He also gives a critical account on the important roll of the consulations at the "Würzburger Synode".

The aim of ecclesiastical effort in this field of communication is the formation of journalists with professional competence, who do not separate between "being a Christian" and „being a journalist", but experience the inner relationship between these "two beings".

RÉSUMÉ: Eglise et journalisme. Un regard en arrière sur les succès et les échecs des dernières décennies

L'auteur nous présente une rétrospective personnelle et retenue de l'histoire du rapport entre l'Eglise et journalisme dans l'époque de l'aprèsguerre en Allemagne Fédérale. Il décrit des impressions tirées de l'histoire compliquée de la fondation de la société appartenant à l'église appelée "Institut zur Förderung publizistischen Nachwuchses e. V." (Institut d'encouragement à la relève journalistique) ainsi que des efforts vains pour la création d'un hebdomadaire important intitulé "public". Les consultations lors de la „Synode de Würzburg" y ont joué un rôle important. L'auteur les commente d'une façon critique.

A l'avenir, il comprend le but de la formation journalistique de l'église comme étant le chrétien engagé avec des compétences professionnelles, car il s'agit que le fait "d'être chretien" et le fait "d'être journaliste" ne soient pas considerés comme des mondes séparés, mais qu'ils soient perçus dans leur appartenance mutelle profonde.

RESUMEN: Iglesia y publicidad. Una mirada retrospectiva a los éxitos y fracasos de las últimas décadas

El autor da una mirada retrospectiva personal a la historia de la relación entre Iglesia y publicidad en el período de la posguerra en la República Federal de Alemania. Nos da a conocer impresiones acerca de la complicada historia inicial de la institución de la Iglesia "Institut zur Förderung des publizistischen Nachwuchses e. V."; también trata del intento frascasado de crear un gran semanario católico que llevaba el nombre de "Publik". En este caso jugó un papel muy importante las deliberaciones en el "Sínodo de Würzburg "que el autor comenta críticamente.

El plantea como meta de la formación periodística de la Iglesia el formar cristianos comprometidos con competencia profesional, pues de lo que se trata es que el "ser cristiano" y el "ser periodista" no sean captados como dos mundos separados, sino que sean vistos en su correspondencia interna. 